\title{
ON FREE PRODUCTS OF COMPLETELY REGULAR SEMIGROUPS
}

\author{
PETER R. JONES
}

(Received 26 July 1991)

Communicated by P. G. Trotter

\begin{abstract}
The free product ${ }^{*} \mathbf{C R} S_{i}$ of an arbitrary family of disjoint completely simple semigroups $\left\{S_{i}\right\}_{i \in I}$, within the variety $\mathbf{C R}$ of completely regular semigroups, is described by means of a theorem generalizing that of Kadourek and Polák for free completely regular semigroups. A notable consequence of the description is that all maximal subgroups of ${ }^{*} \mathrm{CR}_{\mathrm{R}} S_{i}$ are free, except for those in the factors $S_{i}$ themselves. The general theorem simplifies in the case of free CR-products of groups and, in particular, free idempotent-generated completely regular semigroups.
\end{abstract}

1991 Mathematics subject classification (Amer. Math. Soc.): primary 20 M 05; secondary 08 A 50.

Based on fundamental insights of Clifford [1], authors such as Gerhard [3], Trotter [9] and Kadourek and Polák [8] have offered solutions to the word problem for the free completely regular semigroup $F \mathbf{C R}_{X}$ on a countably infinite set $X$. This semigroup is clearly the free product, in the variety $\mathbf{C R}$ of completely regular semigroups, of a family of infinite cyclic groups. We prove (Theorem 4.1) that, suitably modified, the method of Kadourek and Polák serves to solve the word problem in the free $\mathbf{C R}$-product of any family of disjoint completely simple semigroups (modulo effective description of the factors themselves). One notable consequence is that the maximal subgroups of such a free product are always free groups, if disjoint from the original factors.

A slight simplication occurs for the free CR-product of groups. In particular,

(C) 1994 Australian Mathematical Society 0263-6115/94 \$A2.00+0.00 
the word problem is solved for the free idempotent-generated completely regular semigroup (which is a free product of trivial groups) on a set $X$. This semigroup turns out to be isomorphic with the completely regular subsemigroup of $F \mathbf{C R}_{X}$ generated by $\left\{x^{0}: x \in X\right\}$.

The general problem, of describing arbitrary free CR-products, seems unassailable at present. See [7] for a review of the current state of affairs on free band products. The free product of completely simple semigroups within the variety of completely simple semigroups was described in [6]. Free products within some other 'small' subvarieties, such as Clifford semigroups and normal bands of groups are also not difficult to describe.

\section{Free CR-products}

A semigroup $S$ is completely regular if it is a union of its maximal subgroups. For an element $x$ of $S, x^{-1}$ and $x^{0}$ denote the inverse of $x$ and the identity element, respectively, in the maximal subgroup $H_{x}$. See $[2,5]$ for basic properties of such semigroups.

Throughout, $\mathbf{U}$ will denote the variety of unary semigroups (semigroups equipped with a unary operation $x \rightarrow x^{-1}$ ). The class $\mathbf{C R}$ of completely regular semigroups forms a (unary) subvariety of $\mathbf{U}$, determined by the identities

$$
x x^{-1} x=x, \quad x x^{-1}=x^{-1} x, \quad\left(x^{-1}\right)^{-1}=x .
$$

Let $\left\{S_{i}\right\}_{i \in I}$ be a family of disjoint completely regular semigroups; denote their free CR-product by $*_{\mathbf{C R}}\left\{S_{i}\right\}_{i \in I}$, or $*_{\mathbf{C R}} S_{i}$ for short. Thus there exist monomorphisms $\eta_{i}: S_{i} \rightarrow *_{\mathrm{CR}} S_{i}, i \in I$, and for any $T \in \mathbf{C R}$ and morphisms $\phi_{i}: S_{i} \rightarrow T$, $i \in I$, there is a unique morphism $\phi: *_{\mathrm{CR}} S_{i} \rightarrow T$ such that $\eta_{i} \phi=\phi_{i}, i \in I$. (See $[4$, Section 9]).

In this section we construct $*_{\mathbf{C R}} S_{i}$ as a quotient of a certain free unary semigroup, which must first be described. For the moment, let $\left\{S_{i}\right\}_{i \in I}$ be any family of disjoint unary semigroups. Let $X=\bigcup_{i \in I} S_{i}$ and let $F$ be the free monoid on the set $X \cup\left\{(,)^{-1}\right\}$. Then (see [3]) $F \mathbf{U}_{X}$ is the smallest subsemigroup of $F$ such that $X \subseteq F \mathbf{U}_{X}$ and $(w)^{-1} \in F \mathbf{U}_{X}$ whenever $w \in F \mathbf{U}_{X}$ (and then, of course, $(w)^{-1}$ is the 'inverse' of $w$ ). Let $\epsilon$ be the unary congruence on $F \mathbf{U}_{X}$ generated by $\left\{(s \cdot t, s t): s, t \in S_{i}, i \in I\right\} \cup\left\{\left((s)^{-1}, s^{-1}\right): s \in S_{i}, i \in I\right\}$, where $s \cdot t$ denotes the product in $F$. The following proposition is easily proved. 
PROPOSITION 1.1. Let $\left\{S_{i}\right\}_{i \in I}$ be a family of disjoint unary semigroups. Put $X=\bigcup_{i \in I} S_{i}$ and let $U=F \mathbf{U}_{X} / \epsilon$. Then $U$ (together with the morphisms $\left.\beta_{i}: x \longrightarrow x \epsilon, x \in S_{i}, i \in I\right)$ is isomorphic with the free $\mathbf{U}$-product $*_{\mathrm{U}} S_{i}$.

The members of the monoid $F$ are words in the letters $X \cup\left\{(,)^{-1}\right\}$. A segment of a word $w$ is a word $s$ such that $w=a s b$ for some $a, b \in F ; s$ is initial if $a=1$ and terminal if $b=1$.

PROPOSITION 1.2. Each $\epsilon$-class of $F \mathbf{U}_{X}$ contains a unique word $w$ such that

(a) successive letters of $w$ do not belong to the same set $S_{i}$ and

(b) $w$ contains no segment of the form $(s)^{-1}$, where $s$ belongs to some $S_{i}$.

PROOF. That each word in $F \mathbf{U}_{X}$ is $\epsilon$-equivalent to such a word is easily established by an inductive argument, based on lengths of words; a simple confluence argument establishes uniqueness.

In the sequel we generally assume, without comment, that $U$ consists of all such words in $F \mathbf{U}_{X}$, with appropriately modified multiplication and inversion. With this understanding we then refer to words, and their letters, in $U$.

PROPOSITION 1.3. Let $\left\{S_{i}\right\}_{i \in I}$ be a family of disjoint completely regular semigroups. Define $\rho$ to be the semigroup congruence on $U=F \mathbf{U}_{X} / \epsilon$ generated by the pairs

$$
\left(u(u)^{-1} u, u\right), \quad\left(u(u)^{-1},(u)^{-1} u\right), \quad\left(\left((u)^{-1}\right)^{-1}, u\right), \quad u \in U .
$$

Then $U / \rho$ is isomorphic with the free $\mathbf{C R}-$ product $*_{\mathrm{CR}} S_{i}$.

PROOF. It is sufficient to show that $\rho$ is a unary congruence, for then the result follows from standard universal algebraic arguments. So suppose $u, v \in U$ and $u \rho=v \rho$. From the generating relations it is clear that $(u)^{-1} \rho$ is an inverse of $u \rho$ in $U / \rho$ and that $(u)^{-1} \rho \mathscr{H} u \rho$ in $U / \rho$. Thus $(u)^{-1} \rho=(u \rho)^{-1}$. Similarly, $(v)^{-1} \rho=(v \rho)^{-1}$ and thus $(u)^{-1} \rho=(v)^{-1} \rho$.

It is well known (and easily proved) that every congruence on a completely regular semigroup is a unary congruence. 


\section{Green's relation $\mathscr{D}$}

Throughout this section, $S$ denotes $U / \rho=*_{\mathbf{C R}} S_{i}$, the free CR-product of arbitrary disjoint completely regular semigroups, and $\beta_{i}$ denotes the injection $S_{i} \longrightarrow S, i \in I$. Green's relation $\mathscr{D}$ on any completely regular semigroup is the least semilattice congruence [5, Theorem 4.6]. The variety $S$ of semilattices is a subvariety of $\mathbf{C R}$. Thus $S / \mathscr{D}$ is isomorphic with the free $\mathbf{S}$-product of the semilattices $Y_{i}=S_{i} / \mathscr{D}, i \in I$. We now describe free $\mathrm{S}$-products.

Let $\left\{Y_{i}\right\}_{i \in I}$ be any family of disjoint semilattices. Let $Y$ be the direct product of the family $\left\{Y_{i}^{(1)}\right\}_{i \in I}$, where $Y_{i}^{(1)}$ is obtained from $Y_{i}$ by adjoining a (new) identity element. Thus $Y$ consists of all functions $f: I \longrightarrow \bigcup_{i \in I} Y_{i}^{(1)}$ with if $\epsilon Y_{i}^{(1)}, i \in I$. The support of such a function $f$ is $\{i \in I:$ if $\neq 1\}$. Let $P$ be the subsemilattice of $Y$ consisting of the functions of finite nonempty support. For each $i \in I$, let $\alpha_{i}: Y_{i} \longrightarrow P$ be defined by

$$
j\left(x \alpha_{i}\right)=\left\{\begin{array}{ll}
x & \text { if } i=j \\
1 & \text { if } i \neq j
\end{array} .\right.
$$

The following is folklore:

RESULT 2.1. The semilattice $P$, with morphisms $\alpha_{i}$ defined above, is isomorphic with the free $\mathbf{S}$-product $*_{\mathrm{S}} Y_{i}$ of the semilattices $Y_{i}, i \in I$.

Now let $u \in U$. We define the content $c(u)$ as a function in $P$ : for $i \in I$, $i c(u)=D_{s(u)}$, where $s(u)$ is the product of all those letters in $u$, if any, that belong to $S_{i}$ (in the order in which they appear, say) or is $1 \in Y_{i}^{(1)}$ if no such letter belongs to $S_{i}$. Clearly the content defines a morphism $c: U \longrightarrow P$. For each $i \in I$ and each $x \in S_{i}, c(x)=c(x \epsilon)=D_{x} \alpha_{i}$, so $\beta_{i} c=\mathscr{D}^{\natural} \alpha_{i}$, where $\mathscr{D}^{\natural}$ is the natural map $S_{i} \longrightarrow Y_{i}$. Thus $c c^{-1}$ is the least semilattice congruence on $U$. Clearly $\rho \subseteq c c^{-1}$, so $c c^{-1}$ induces the least semilattice congruence on $S=U / \rho$. The next proposition therefore describes $\mathscr{D}$ on $S$.

Proposition 2.2. Let $u, v \in U$. Then $u \rho \mathscr{D} v \rho$ in $*_{\mathrm{CR}} S_{i}$ if and only if $c(u)=c(v)$. 


\section{Coproducts of completely simple semigroups: Green's relations}

For the remainder of the paper we specialize to the case where each factor $S_{i}$ belongs to $\mathbf{C S}$, the subvariety of $\mathbf{C R}$ consisting of all completely simple semigroups. At the end of this section we consider some difficulties associated with the general case.

Since, now, each $\left|Y_{i}\right|=\left|S_{i} / \mathscr{D}\right|=1$, we may redefine $c(u)$ as $\left\{i \in I: S_{i}\right.$ contains a letter of $u$ \}; $c$ now defines a morphism of $U$ upon the free semilattice on $I$, consisting of the nonempty finite subsets of $I$, under union.

For each $i \in I$, specify an arbitrary idempotent $s_{i}$ of $S_{i}$. Denote by $L^{(i)}, R^{(i)}$ and $H^{(i)}$, respectively, the $\mathscr{L}$-, $\mathscr{R}$ - and $\mathscr{H}$-class of $s_{i}$ in $S_{i}$. (These play a 'normalizing' role.) For any word $w \in F, \hat{w}$ denotes the word of $U^{1}$ obtained from $w$ by deleting all unmatched parentheses and choosing the representative of the resulting $\epsilon$-class (see Section 1).

Let $u \in U$, with content $A$, say, $|A|=n \geq 1$. By analogy with [1,3,9], put $u 0=\hat{a}$, where $a$ is the longest initial segment of $u$ such that $c(a) \neq A$. Let $y$ be the next letter of $u$ after the segment $a$; then $y \notin\left\{(,)^{-1}\right\}$, so $y \in S_{j}$, where $A-c(u 0)=\{j\}$. Put $u \sigma=\left(y s_{j}\right)^{0}$, the idempotent in the $\mathscr{H}$-class $R_{y} \cap L^{(j)}$ of $S_{j}$. Thus $y=(u \sigma) y$ and $u 0 y=u 0 u \sigma \quad y$. The product $L(u)=u 0 u \sigma$ is defined to be the left indicator of $u$.

Dually, let $u 1=\hat{b}$, where $b$ is the longest terminal segment of $u$ such that $c(b) \neq A$; let $u \epsilon=\left(s_{j} x\right)^{0}$, the idempotent in the $\mathscr{H}$-class $L_{x} \cap R^{(k)}$, where $x \in S_{k}$ is the last letter of $u$ before the segment $b$, and put $R(u)=u \epsilon u 1$, the right indicator of $u$. Note that if $c(u)=\{i\}$, that is, $u$ belongs to the factor $S_{i}$, then $u 0=1=u 1$ and $u \sigma$ and $u \epsilon$ are, respectively, the idempotents $\left(u s_{i}\right)^{0}$ and $\left(s_{i} u\right)^{0}$ of $S_{i}$.

LEMMA 3.1. Let $u, v \in U$, with $u \rho v$. If

(i) $|c(u)|=1$ then $u=v$;

(ii) $|c(u)|>1$ then $u 0 \rho v 0$ and $u \sigma=v \sigma$, and dually.

Proof. (i) Suppose $u \in S_{i}, i \in I$. By Proposition 2.2, $c(u)=c(v)$, so $v \in S_{i}$ also. Since $S_{i}$ embeds in $S, u=v$.

(ii) It suffices to prove the result when $v$ is obtained from $u$ by an elementary transition. Let $u_{1}$ be the shortest initial segment of $u$ with content that of $u$. In the notation above, $u_{1}=a y$ and $\hat{u}_{1}=\hat{a} y=u 0 y$. In the free monoid $F, u=u_{1} u_{2}$ 
for some $u_{2}$.

Suppose $u$ factors as $p q r$, in $U$, with $p$ and/or $q$ possibly 1 ; note that all matched pairs of parentheses lie within $p, q$ or $r$. Let $v=p q(q)^{-1} q r$. Various possibilities must be treated.

(a) If $a=p q r_{1}$ and $r=r_{1} y u_{2}$ in $U$ (where $r_{1}$ may be 1) then $u=p q r_{1} y u_{2}$ and $v=\left(p q(q)^{-1} q r_{1}\right) y u_{2}$; now $v 0=\left(p q(q)^{-1} q r_{1}\right)^{\wedge}=p q(q)^{-1} q \hat{r}_{1} \rho p q \hat{r}_{1}=$ $\left(p q r_{1}\right)^{\wedge}=u 0$. Clearly, $u \sigma=v \sigma$.

(b) If $a=p q_{1}, y=q_{2} r_{1}, q=q_{1} q_{2}$ and $r=r_{1} u_{2}$, where $q_{1}, r_{1}$ may be 1 , then $u=p q_{1} q_{2} r_{1} u_{2}$ and $v=p q_{1} q_{2}\left(q_{1} q_{2}\right)^{-1} q_{1} q_{2} r_{1} u_{2}$. Now $c\left(q_{2}\right)=c(y)$, so $v 0=\left(p q_{1}\right)^{n}=u 0$. Also $y=q_{2} r_{1} \mathscr{R} q_{2}$ (within some completely simple factor), so $v \sigma=u \sigma$.

(c) In all other cases of this transition, $v$ and $u$ both begin with the segment ay. Thus $v 0=u 0$ and $v \sigma=u \sigma$.

The reverse transition is handled similarly. Transitions associated with $(q)^{-1} q \longrightarrow q(q)^{-1}$ and $q \longrightarrow\left(q^{-1}\right)^{-1}$ and their reverses involve only addition or deletion of parentheses and are handled easily.

LEMMA 3.2. Let $u \in U,|c(u)|>1$. Let $u_{1}$ be an initial segment of $u$, regarded as a word in $F$. Then $u \rho \hat{u}_{1} u_{2}$, for some $u_{2} \in U$. Thus $u \rho \hat{u}_{1}\left(\hat{u}_{1}\right)^{-1} u$ and, in particular, $u \rho L(u) u^{\prime}$ for some $u^{\prime} \in U$.

PROOF. We proceed by induction on the number of unmatched left parentheses in $u_{1}$. Let $u=u_{1} w$ in $F$. If $u_{1}$ has no unmatched parenthesis then $\hat{u}=\hat{u}_{1} \hat{w}$ (as a product in $U)$. Otherwise, write $u_{1}=p(q$, where $q$ has no unmatched left parenthesis. Since $u \in U, w=r)^{-1} s$ for some $r, s$. Thus $u=p(q r)^{-1} s$ in $U$, so $u \rho v=p q r(q r)^{-1}(q r)^{-1} s$. But $p q$ has one fewer unmatched left parenthesis, so $v \rho(p q)^{\wedge} u_{2}$ for some $u_{2} \in U$, where $(p q)^{\wedge}=\hat{u}_{1}$. This completes the proof of the first statement, the second being an immediate consequence. To prove the third, we use the notation in the preamble to Lemma 3.1: $L(u)=u 0 u \sigma=$ $\hat{a} g=(a g)^{\wedge}$, where $u=a y s \epsilon a g \cdot y s$, so that $a g$ is an initial segment of $u$, in $F$.

For ease of exposition, we extend $\rho$ from $U$ to $U^{1}$ by putting $1 \rho=\{1\}$.

THEOREM 3.3. Let $\left\{S_{i}\right\}_{i \in I}$ be a family of disjoint completely simple semigroups, $U=*_{\mathrm{U}} S_{i}$ and $S=U / \rho=*_{\mathrm{CR}} S_{i}$. If $u, v \in U$ then

(i) $\quad u \rho \mathscr{D} v \rho$ if and only if $c(u)=c(v)$; 
(ii) $u \rho \mathscr{R} v \rho$ if and only if $u 0 \rho v 0$ and $u \sigma=v \sigma$;

(iii) $u \rho \mathscr{L} v \rho$ if and only if $u 1 \rho v 1$ and $u \epsilon=v \epsilon$.

\section{PROOF.}

(i) In view of the opening remarks of this section, this is a special case of Proposition 2.2.

(ii) We first observe that $u \rho \mathscr{R} L(u) \rho$. For by the preceding lemma, $\mathscr{R}_{u \rho} \leq R_{L(u) \rho}$; but $L(u) \rho \mathscr{D} u \rho$ in $S$, since $c(L(u))=c(u)$, so equality of $\mathscr{R}$ classes holds, the $\mathscr{D}$-class being completely simple. As a consequence, $u \rho \mathscr{R} v \rho$ if and only if $L(u) \rho \mathscr{R} L(v) \rho$. One implication is then immediate. Conversely, if $L(u) \rho \mathscr{R} L(v) \rho$ then $u 0 u \sigma \rho v 0 v \sigma t$ for some $t \in U$. But $(v 0 v \sigma t) 0=v 0$ and $(v 0 v \sigma t) \sigma=v \sigma$ (since if $t$ begins with a letter from the same factor as $v \sigma$, then $v \sigma t \mathscr{R} v \sigma$ and the definitions of $(v 0 v \sigma) \sigma$ and $(v 0 v \sigma t) \sigma$ yield $\mathscr{H}$-related idempotents). By Lemma 3.1, $u 0 \rho v 0$ and $u \sigma=v \sigma$.

(iii) This is dual to (ii).

This result simplifies considerably when the factors are groups-see Section 5.

The proofs of the results in this section have followed closely those of Clifford for free completely regular semigroups [1]. The author [7] has modified these techniques to obtain some properties of free products of bands, in the variety $\mathbf{B}$ of all bands. The difficulties which arise when the factors are no longer completely simple are discussed in detail there. We conclude this section with an example to show that in that case Green's relations $\mathscr{R}$ and $\mathscr{L}$ are not determined in such a simple fashion.

Let $S_{1}$ be a trivial semigroup $\{e\}$ and let $S_{2}$ be a two-element semilattice $\{f, g\}, f>g$. In $U, e g=e f g \rho e f(e f)^{-1} e f g=e f(e f)^{-1} e g$. Suppose we were to define $u 0$ as $\hat{a}$, where $a$ is the longest initial segment of $u$ involving all the factors but one that appear in $u$, and define $u \sigma$ as above. Then $(e g) 0=e$ and $(e g) \sigma=g$; also $\left(e f(e f)^{-1} e g\right) 0=e$ and $\left(e f(e f)^{-1} e g\right) \sigma=f$. Thus Lemma 3.1 would now fail.

A slightly less naive definition for $u 0$ would be as $\hat{a}$, with $a$ the longest initial segment whose content is greater than that of $u$ (using the general definition of content in Section 2) and with $u \sigma$ as before. In the given example, $(e g) 0=e$ but $\left(e f(e f)^{-1} e g\right) 0=e f(e f)^{-1} e$. Since $e$ and $e f(e f)^{-1} e$ have different contents they cannot be $\rho$-related. So Lemma 3.1 again fails. Nevertheless, some positive results have been obtained for free band products [7], leaving open the prospect 
of further progress for completely regular semigroups in general.

\section{Coproducts of completely simple semigroups: the word problem}

Let $\left\{S_{i}\right\}_{i \in I}$ be a family of disjoint completely simple semigroups; define $U=*_{\mathrm{U}} S_{i}$, recalling the remarks following Proposition 1.2; and let $S=U / \rho=$ $*_{\mathrm{CR}} S_{i}$, as in Section 1. We solve the word problem in $S$ (modulo those in the factors $S_{i}$ ) inductively, based on $|c(u)|$, that is, on the number of factors $S_{i}$ involved in the word $u \in U$ under consideration.

If $|c(u)|=1$ and $u \rho v$ then $u, v \in S_{i}$ for some $i \in I$ and so $u=v$ in $S_{i}$ (since $S_{i}$ embeds in $S$ ). The inductive step involves an extension of the notion of characteristic sequence introduced in [8].

Let $|c(u)|=n \geq 2$. The characteristic sequence $[u]$ of $u$, to be constructed below, will have the form

$$
\left(\mu_{j} e_{j} u_{j} g_{j}\right)_{0 \leq j \leq k}=\left(\mu_{0} e_{0} u_{0} g_{0}, \mu_{1} e_{1} u_{1} g_{1}, \ldots, \mu_{k} e_{k} u_{k} g_{k}\right)
$$

where for each $j, \mu_{j} \in\{1,-1\}, c\left(u_{j}\right)=c(u)-\left\{i_{j}\right\}$ for some $i_{j} \in c(u)$ and $e_{j}$ and $g_{j}$ are idempotents of $S_{i_{j}}^{1}$. The expression $e_{j} u_{j} g_{j}$ is to be regarded as a product in $U$ (or in $F$ ); it is termed a link of $u$. (This term has a more general meaning here than as originally used in [9]). A link $e_{j} u_{j} g_{j}$ is interior if neither $e_{j}$ nor $g_{j}$ is 1 . Let Link $(u)$ be the set of interior links of $u$. It will be shown that Link $(u)=\left\{e_{j} u_{j} g_{j}: 0<j<k\right\}$ and that $e_{0} u_{0} g_{0}$ and $e_{k} u_{k} g_{k}$ are, respectively, the left and right indicators of $u$.

The characteristic sequence is constructed inductively, as in [8], on the number of segments $(q)^{-1}$ of $u$ with $c(q)=c(u)$.

(i) Suppose $u$ has no such segment. Let $b_{0}, \ldots, b_{k}$ be the sequence of segments of $u$, read from left to right, that are maximal such that $\left|c\left(b_{j}\right)\right|=$ $|c(u)|-1$. Put $u_{j}=\hat{b}_{j}, 0 \leq j \leq k$. Let $c(u)-c\left(b_{j}\right)=\left\{i_{j}\right\}$ and let $x_{j}$ and $y_{j}$, respectively, be the letters of $u$ that immediately precede and follow $b_{j}$ (with value 1 if empty). Let $e_{j}=\left(s_{i_{j}} x_{j}\right)^{0}$ and $g_{j}=\left(y_{j} s_{i_{j}}\right)^{0}$, the idempotent in the $\mathscr{H}$-class $R^{\left(i_{j}\right)} \cap L_{x_{j}}$ or $L^{\left(i_{j}\right)} \cap R_{y_{j}}$, respectively, of $S_{i_{j}}$; or 1 if $x_{j}=1$ or $y_{j}=1$. Let $[u]$ be the sequence $\left(+e_{j} u_{j} g_{j}\right)_{0 \leq j \leq k}$ so defined.

(ii) Now suppose $u=p(q)^{-1} r$, where $c(q)=c(u)$. Put

$$
[u]=\left(\langle p q 0\rangle_{q \sigma},-{ }_{q \epsilon}[q 1 q q 0]_{q \sigma},{ }_{q \epsilon}\langle q 1 r\rangle\right)
$$




\begin{tabular}{|l|l|}
\hline$R^{(1)}$ & $b$ \\
\hline$a$ & $c$ \\
\hline
\end{tabular}

$L^{(1)}$

$S_{1}$
$R^{(2)}$

\begin{tabular}{|l|l|}
\hline$f$ & $j$ \\
\hline$g$ & $h$ \\
\hline
\end{tabular}

$L^{(2)}$

FIGURE 1

where

$$
\begin{aligned}
\langle w\rangle & =\left\{\begin{array}{ll}
1 \cdot w \cdot 1 & \text { if }|c(w)|<|c(u)| \\
{[w]} & \text { if }|c(w)|=|c(u)|
\end{array},\right. \\
& -\left(w_{0}, \ldots, w_{\ell}\right)=\left(-w_{\ell}, \ldots,-w_{0}\right),
\end{aligned}
$$

and pre-subscripting [post-subscripting] denotes pre-multiplication [post-multiplication] of the first [last] link by the subscript.

In case (i) it is clear from a comparison of the definitions that $\mu_{0} e_{0} u_{0} g_{0}$ is the left indicator of $u$, that is, $\mu_{0}=1, e_{0}=1, u_{0}=u 0$ and $g_{0}=u \sigma$. In fact, a simple induction establishes that this is true for any $u$. Similarly, $\mu_{k} e_{k} u_{k} g_{k}$ is the right indicator of $u$, that is, $\mu_{k}=1, e_{k}=u \epsilon, u_{k}=u 1$ and $g_{k}=1$.

EXAMPLE. Let $S_{1}$ and $S_{2}$ be the two rectangular bands defined in Figure 1, with designated idempotents $s_{1}=e, s_{2}=f$ and the designated $\mathscr{L}$ - and $\mathscr{R}$-classes thus as indicated.

Consider the word $u=a j b(a j b)^{-1} a f \in U$. Then $(a j b) 0=a,(a j b) \sigma=$ $(j f)^{0}=f,(a j b) 1=b$ and $(a j b) \epsilon=(f j)^{0}=j$; note that $(a j b) a=a j(b a)=$ aje; similarly $b(a j b)=e j b$. Thus

$$
[u]=\left(\langle a j e\rangle_{f},-_{j}[e j e]_{f},{ }_{j}\langle e f\rangle\right) .
$$

Now $c(a j e)=c(u)$, so $\langle a j e\rangle=[a j e]=(1 a f, e j e, j e \cdot 1)$ and $\langle a j e\rangle_{f}=$ $(1 a f, e j e, j e f)$. Similarly, ${ }_{j}\langle e f\rangle=(j e f, e f \cdot 1)$. Also $[e j e]=(1 e f, e j e, j e$. $1)$, so ${ }_{j}[e j e]_{f}=(j e f, e j e, j e f)$ and $-{ }_{j}[e j e]_{f}=(-j e f,-e j e,-j e f)$. Hence

$$
[u]=(1 a f, e j e, j e f,-j e f,-e j e,-j e f, j e f, \text { ef } \cdot 1) .
$$


Similarly,

$$
\begin{aligned}
{\left[(a j b)^{-1} \text { aj ef }\right] } & =\left(\langle a\rangle_{f},-{ }_{j}[e j e]_{j},{ }_{j}[\text { ejef }]\right) \\
& =(1 a f,-j e f,-e j e,-j e f, \text { jef, eje, jef, ef } \cdot 1) .
\end{aligned}
$$

Let $Z$ be a countably infinite set of new variables and let $F \mathbf{G}_{Z}$ denote the free group on $Z$. Our main theorem is a close analogue of the Theorem in [8].

THEOREM 4.1. Let $\left\{S_{i}\right\}_{i \in I}$ be a family of disjoint completely simple semigroups, let $U=*_{\mathrm{U}} S_{i}$ and $S=U / \rho=*_{\mathrm{CR}} S_{i}$. Let $u, v \in U$. If $|c(u)|=1$ then $u \rho v$ if and only $u=v$; if $|c(u)|>1$ then $u \rho v$ if and only if

(i) $c(u)=c(v)$;

(ii) $u 0 \rho v 0$ and $u \sigma=v \sigma$;

(iii) $u 1 \rho v 1$ and $u \epsilon=v \epsilon$;

(iv) if $[u]=\left(\mu_{j} e_{j} u_{j} g_{j}\right)_{0 \leq j \leq k}$ and $[v]=\left(\eta_{j} f_{j} v_{j} h_{j}\right)_{0 \leq j \leq \ell}$ and $\psi$ is any map from Link $(u) \cup \operatorname{Link}(v)$ into $Z$ such that $(e w g) \psi=\left(e^{\prime} w^{\prime} g^{\prime}\right) \psi$ if and only if $e=e^{\prime}, w \rho w^{\prime}$ and $g=g^{\prime}$, then

$$
\prod_{j=1}^{k}\left(\left(e_{j} u_{j} g_{j}\right) \psi\right)^{\mu_{j}}=\prod_{j=1}^{\ell}\left(\left(f_{j} v_{j} h_{j}\right) \psi\right)^{\eta_{j}} \quad \text { in } F \mathbf{G}_{Z} .
$$

If Link $(u)$ or Link $(v)$ is empty, the product is interpreted as the identity element of $F \mathbf{G}_{Z}$. Before its proof, the theorem will be exemplified.

EXAMPLE. In the example above, Link $\left(a j b(a j b)^{-1} a f\right)=\{e j e, j e f\}=$ Link $\left((a j b)^{-1}\right.$ ajef $)$. We may map eje to $z_{1}$ and jef to $z_{2}$, say, $z_{1} \neq z_{2}$. Then the product associated with $a j b(a j b)^{-1}$ af is $z_{1} z_{2} z_{2}^{-1} z_{1}^{-1} z_{2}^{-1} z_{2}=1$ and that with $(a j b)^{-1}$ ajef is $z_{2}^{-1} z_{1}^{-1} z_{2}^{-1} z_{2} z_{1} z_{2}=1$. According to Theorem 4.1, therefore, $a j b(a j b)^{-1}$ af and $(a j b)^{-1}$ ajef represent the same element of $S$.

PROOF OF NECESSITY. Necessity of (i)-(iii) follows from Theorem 3.3. From the definition of $\rho$, to prove (iv) it is only necessary to show that

(a) (iv) holds for $\left(u(u)^{-1} u, u\right),\left(u(u)^{-1},(u)^{-1} u\right)$ and $\left(\left((u)^{-1}\right)^{-1}, u\right)$ for all $u \in U$;

(b) if $u \rho v$ and (iv) holds for $(u, v)$ then (iv) holds for (su,sv) and (us, vs), for any $s \in U$. 
We need the following lemma. Observe first that the operators 0 and $\sigma$ on words in $U$ may be iterated: put $u 0^{0}=u$ and $u \sigma^{0}=1$ and define $u 0^{n}=\left(u 0^{n-1}\right) 0$ and $u \sigma^{n}=\left(u 0^{n-1}\right) \sigma$ for $n \geq 1$. The operators 1 and $\epsilon$ are iterated similarly.

LEMMA 4.2. Let $s, u \in U$. Then

(i) if $c(s)$ and $c(u)$ are incomparable then $[s u]=\left(+e_{j} w_{j} g_{j}\right)_{0 \leq j \leq h}$, where $e_{j}=s \epsilon^{j \alpha}, w_{j}=s 1^{j \alpha} u 0^{j \beta}, g_{j}=u \sigma^{j \beta}, 0 \alpha=h \beta=0$ and for $0<j<h, j \alpha$ and $j \beta$ are positive integers determined by the order in which the factors $S_{i}$ involved in s last appear and the order in which the factors involved in $u$ first appear, respectively;

(ii) if $c(u) \subseteq c(s)$ and $[s]=\left(\eta_{j} f_{j} s_{j} h_{j}\right)_{0 \leq j \leq \ell}$ then

$$
[s u]=\left(\eta_{0} f_{0} s_{0} h_{0}, \ldots, \eta_{\ell-1} f_{\ell-1} s_{\ell-1} h_{\ell-1}, f_{\ell}\left\langle s_{\ell} u\right\rangle\right) .
$$

PROOF. This follows straightforwardly from the definition of characteristic sequence.

To prove (a) above, let $u \in U,|c(u)| \geq 2$, and suppose $[u]=\left(\mu_{j} e_{j} u_{j} g_{j}\right)_{0 \leq j \leq k}$ $=\left(1 u_{0} g_{0}, T, e_{k} u_{k} 1\right)$, say. By definition, recalling that $u_{0}=u 0, g_{0}=u \sigma$, $e_{k}=u \epsilon$ and $u_{k}=u 1$,

$$
\left[u(u)^{-1} u\right]=\left(\left[u u_{0}\right]_{g_{0}},-e_{e_{k}}\left[u_{k} u u_{0}\right]_{g_{0}}, e_{k}\left[u_{k} u\right]\right) .
$$

By Lemma 4.2 (ii) and its dual,

$$
\begin{aligned}
{\left[u u_{0}\right]_{g_{0}} } & =\left(1 u_{0} g_{0}, T, e_{e_{k}}\left\langle u_{k} u_{0}\right\rangle_{g_{0}}\right), \\
e_{k}\left[u_{k} u\right] & =\left(e_{k}\left\langle u_{k} u_{0}\right\rangle_{g_{0}}, T, e_{k} u_{k} \cdot 1\right) \quad \text { and } \\
e_{k}\left[u_{k} u u_{0}\right]_{g_{0}} & =\left(e_{e_{k}}\left\langle u_{k} u_{0}\right\rangle_{g_{0}}, T, e_{e_{k}}\left\langle u_{k} u_{0}\right\rangle_{g_{0}}\right) .
\end{aligned}
$$

Substituting these three sequences into the previous one, applying an appropriate map $\psi$ and evaluating in $F \mathbf{G}_{Z}$ verifies that (iv) holds in this case. The case $\left(u(u)^{-1},(u)^{-1} u\right)$ is similar. Considering $\left(\left((u)^{-1}\right)^{-1}, u\right)$ we have, applying the definition,

$$
\begin{aligned}
{\left[\left((u)^{-1}\right)^{-1}\right] } & =\left(\left\langle u_{0}\right\rangle_{g_{0}},-{ }_{e_{k}}\left[u_{k}(u)^{-1} u_{0}\right]_{g_{0}}, e_{k}\left\langle u_{k}\right\rangle\right) \\
& =\left(1 u_{0} g_{0},-{ }_{e_{k}}\left[u_{k}(u)^{-1} u_{0}\right]_{g_{0}}, e_{k} g_{k} \cdot 1\right) .
\end{aligned}
$$

Applying the definition once more,

$$
\left[u_{k}(u)^{-1} u_{0}\right]=\left(\left\langle u_{k} u_{0}\right\rangle_{g_{0}},--_{e_{k}}\left[u_{k} u u_{0}\right]_{g_{0}}, e_{k}\left\langle u_{k} u_{0}\right\rangle\right)
$$


and so $e_{k}\left[u_{k}(u)^{-1} u_{0}\right]_{g_{0}}=\left(e_{e_{k}}\left\langle u_{k} u_{0}\right\rangle_{g_{0}},-_{e_{k}}\left[u_{k} u u_{0}\right]_{g_{0}}, e_{e_{k}}\left\langle u_{k} u_{0}\right\rangle_{g_{0}}\right)$. Substituting this last sequence in the expression above and using the formulas obtained for the first case, we obtain

$$
\left[\left((u)^{-}\right)^{-1}\right]=\left(1 u_{0} g_{0},-e_{e_{k}}\left\langle u_{k} u_{0}\right\rangle_{g_{0}}, e_{k}\left\langle u_{k} u_{0}\right\rangle_{g_{0}}, T, e_{e_{k}}\left\langle u_{k} u_{0}\right\rangle_{g_{0}},-_{e_{k}}\left\langle u_{k} u_{0}\right\rangle_{g_{0}}, e_{k} g_{k} \cdot 1\right)
$$

The proof may now be easily completed.

The proof of (b) above is by induction on the content. Suppose that (iv) holds for $(u, v), u, v \in U, u \rho v$, and let $s \in S$. By Theorem 3.3, $c(u)=c(v)$. Suppose firstly that $c(u)$ and $c(s)$ are incomparable. Then $[s u]=\left(+e_{j} w_{j} g_{j}\right)_{0 \leq j \leq h}$, in the notation of Lemma 4.2 (i). Similarly, $[s v]=\left(+f_{j} z_{j} h_{j}\right)_{0 \leq j \leq \ell}$, where by repeated applications of Lemma 3.1, from $u \rho v$ it follows that $h=\ell$, that $f_{j}=s 1^{j \alpha}=e_{j}$ and $h_{j}=v \sigma^{j \beta}=u \sigma^{j \beta}=g_{j}$, and that $z_{j}=s 1^{j \alpha} v 0^{j \beta} \rho$ $s 1^{j \alpha} u 0^{j \beta}=w_{j}$, for $0 \leq j \leq h$. Thus for any appropriate map $\psi$ into $Z$, $\left(e_{j} w_{j} g_{j}\right) \psi=\left(f_{j} z_{j} h_{j}\right) \psi, 0<\bar{j}<h$, and so $\prod_{j=1}^{h-1}\left(e_{j} w_{j} g_{j}\right) \psi=\prod_{j=1}^{h-1}\left(f_{j} z_{j} h_{j}\right) \psi$ in $F \mathbf{G}_{Z}$, that is, (iv) holds for (su,sv).

Next suppose $c(u) \subseteq c(s)$. Let $[s]=\left(\eta_{j} f_{j} s_{j} h_{j}\right)_{0 \leq j \leq \ell}=\left(1 s_{0} h_{0}, S, f_{\ell} s_{\ell} \cdot 1\right)$, say. By Lemma 4.2 (ii),

$$
\begin{aligned}
& {[s u]=\left(1 s_{0} h_{0}, S, f_{\ell}\left\langle s_{\ell} u\right\rangle\right) \quad \text { and }} \\
& {[s v]=\left(1 s_{0} h_{0}, S, f_{\ell}\left\langle s_{\ell} v\right\rangle\right) .}
\end{aligned}
$$

If $c(u) \subseteq c\left(s_{\ell}\right)$ then ${ }_{f_{\ell}}\left\langle s_{\ell} u\right\rangle=f_{\ell}\left(s_{\ell} u\right) \cdot 1$ and $f_{f_{\ell}}\left\langle s_{\ell} v\right\rangle=f_{\ell}\left(s_{\ell} v\right) \cdot 1$. Since $\psi$ is only applied to interior links, the result is clear. Otherwise, $c\left(s_{\ell} u\right)=c(s u)$ and $\left\langle s_{\ell} u\right\rangle=\left[s_{\ell} u\right],\left\langle s_{\ell} v\right\rangle=\left[s_{\ell} v\right]$. If $c(u)$ and $c\left(s_{\ell}\right)$ are incomparable then the first part of the proof shows that (iv) holds for $\left(s_{\ell} u, s_{\ell} v\right)$. Moreover, the leading terms of $f_{\ell}\left[s_{\ell} u\right]$ and $f_{\ell}\left[s_{\ell} v\right]$ are $\rho$-related since they are, respectively, $f_{\ell}\left(s_{\ell} u\right) 0\left(s_{\ell} u\right) \sigma$ and $f_{\ell}\left(s_{\ell} v\right) 0\left(s_{\ell} v\right) \sigma$ and applying Lemma 3.1 to $s_{\ell} u$ and $s_{\ell} v,\left(s_{\ell} u\right) 0 \rho\left(s_{\ell} v\right) 0$ and $\left(s_{\ell} u\right) \sigma=\left(s_{\ell} v\right) \sigma$. Under an appropriate map $\psi$, these two terms are therefore equal and (iv) holds for $(s u, s v)$. The remaining case has $c\left(s_{\ell}\right) \subsetneq c(u)$ (that is, $c(u)=c(s))$. By the dual of Lemma 4.2(ii), $f_{\ell}\left[s_{\ell} u\right]=\left(f_{\ell}\left\langle s_{\ell} u 0\right\rangle_{u \sigma}, T, u \epsilon(u 1) \cdot 1\right)$ and $f_{f_{\ell}}\left[s_{\ell} v\right]=\left(f_{\ell}\left\langle s_{\ell} v 0\right\rangle_{v \sigma}, V, v \epsilon(v 1) \cdot 1\right)$, where $[u]=(1 u 0 u \sigma, T, u \epsilon(u 1) \cdot 1)$ and $[v]=(1 v 0 v \sigma, V, v \epsilon(v 1) \cdot 1)$. The case where $c\left(s_{\ell}\right)$ and $c(u 0)$ are incomparable is completed similarly to the previously considered case. In the remaining possibility, $f_{\ell}\left\langle s_{\ell} u 0\right\rangle_{u \sigma}=f_{\ell}\left(s_{\ell} u 0\right) u \sigma$ and $f_{\ell}\left\langle s_{\ell} v 0\right\rangle_{v \sigma}=f_{\ell}\left(s_{\ell} v 0\right) v \sigma$. As above, these links are $\rho$-related and therefore equal under any appropriate map $\psi$. It now follows that (iv) holds for $(s u, s v)$ in this case also.

The alternative case, $c(s) \subseteq c(u)$, holds similarly. Thus (iv) holds for $(s u, s v)$ in all cases. By duality, it holds for (us, vs) also. 
PROOF OF SUFFICIENCY. The main tool in the proof of sufficiency in [8] was the 'Decomposition Lemma,' based on arguments in [9]. Its analogue here is Lemma 4.3. Recall from Section 3 that for $i \in I, s_{i}$ denotes the distinguished idempotent of $S_{i}$, belonging to the $\mathscr{H}$-class $H^{(i)}=R^{(i)} \cap L^{(i)}$. It will be convenient to assume $I$ is well ordered.

LEMMA 4.3. Let $u \in U, c(u)=A$, say, $|A| \geq 2$. Let $[u]=\left(\mu_{j} e_{j} u_{j} g_{j}\right)_{0 \leq j \leq k}$. Then

$$
u \rho \omega_{L}\left(1 u_{0} g_{0}\right) \prod_{j=1}^{k-1} \omega\left(e_{j} u_{j} g_{j}\right)^{\mu_{j}} \omega_{R}\left(e_{k} u_{k}\right)
$$

where for ewg $\in\left\{e_{j} u_{j} g_{j}: 0 \leq j \leq k\right\}$,

$$
\begin{aligned}
\omega_{L}(e w g) & =e w g f(f R(w) g f)^{-1} \\
\omega(e w g) & =f e w g f(f R(w) g f)^{-1} \\
\omega_{R}(e w g) & =f e w g
\end{aligned}
$$

and if $A=\left\{i_{1}<\ldots<i_{n}\right\}, f=s_{i_{1}} \ldots s_{i_{n}}\left(s_{i_{1}} \ldots s_{i_{n}}\right)^{-1} \in U$.

PROOF. For notational convenience, put $I_{j}=R\left(u_{j}\right) g_{j}\left(=u_{j} \epsilon u_{j} 1 g_{j}\right), 0 \leq$ $j<k$. Observe that $I_{j}$ also equals $e_{j+1} L\left(u_{j+1}\right)=e_{j+1} u_{j+1} 0 u_{j+1} \sigma$.

First consider the case where $u$ has no segment $(q)^{-1}$ with $c(q)=c(u)$. Let $d$ denote the terminal segment of $u$ that begins with $u_{1}$ and put $W=\hat{d}$. If, on the one hand, $u_{0}$ was obtained from the initial segment $b_{0}$ (see the definition of $[u])$ without deleting any unmatched parentheses then $u=u_{0} u^{\prime}=u_{0} g_{0} u^{\prime}$, for some $u^{\prime} \in U$; thus $W=\left(u_{0} 1\right) u^{\prime}=\left(u_{0} 1\right) g_{0} u^{\prime}$. By the dual of Lemma 3.2, $u_{0} g_{0} \rho u_{0} g_{0}\left(I_{0}\right)^{-1} I_{0}=u_{0} g_{0}\left(I_{0}\right)^{-1} R\left(u_{0}\right) g_{0}=u_{0} g_{0}\left(I_{0}\right)^{-1} e_{1}\left(u_{0} 1\right) g_{0}$. Therefore $u \rho u_{0} g_{0}\left(I_{0}\right)^{-1} e_{1} W$.

On the other hand, if some parenthesis was deleted from $b_{0}$ we may write $u_{0}=p \hat{g}$, where $g$ begins with a left parenthesis and is an initial segment of some segment $(e)^{-1}$ of $u$. So $u=p(e)^{-1} f$ for some $f \in U^{\prime}$. Since, by hypothesis, $c(e) \neq c(u)$, and the right parenthesis $)^{-1}$ is to the right of $g_{0}=u \sigma$ (see Section 3), it follows that the initial ( of $g$ is to the right of $(u 0) \epsilon$, whence $u_{0} 1=h \hat{g}$ for some $h \in U^{1}$. Thus $W=h(e)^{-1} f$ and since by Lemma 3.2, $(e)^{-1} \rho \hat{g} g_{0}\left(\hat{g} g_{0}\right)^{-1}(e)^{-1}, W \rho h \hat{g} g_{0}\left(\hat{g} g_{0}\right)^{-1}(e)^{-1} f=\left(u_{0} 1\right) g_{0}\left(\hat{g} g_{0}\right)^{-1}(e)^{-1} f$. Similarly

$$
\begin{gathered}
u=p(e)^{-1} f \rho p \hat{g} g_{0}\left(\hat{g} g_{0}\right)^{-1}(e)^{-1} f=u_{0} g_{0}\left(\hat{g} g_{0}\right)^{-1}(e)^{-1} f \\
\rho u_{0} g_{0}\left(I_{0}\right)^{-1} e_{1}\left(u_{0} 1\right) g_{0}\left(\hat{g} g_{0}\right)^{-1}(e)^{-1} f
\end{gathered}
$$




$$
\begin{aligned}
& \rho u_{0} g_{0}\left(I_{0}\right)^{-1} e_{1} h \hat{g} g_{0}\left(\hat{g} g_{0}\right)^{-1}(e)^{-1} f \\
& \rho u_{0} g_{0}\left(I_{0}\right)^{-1} e_{1} W
\end{aligned}
$$

using the proof of the first case in the central step.

In either case, therefore, $u \rho u_{0} g_{0}\left(I_{0}\right)^{-1} e_{1} W$. Next observe that $\left(f\left(f I_{0} f\right)^{-1}\right.$ $\left.f I_{0}\right) \rho$ is an idempotent in $U / \rho$ which is $\mathscr{L}$-related to $\left(u_{0} g_{0}\right) \rho$, by Theorem 3.3, since $I_{0}=R\left(u_{0} g_{0}\right)$. Thus

$$
\begin{aligned}
& u \rho u_{0} g_{0} f\left(f I_{0} f\right)^{-1} f I_{0}\left(I_{0}\right)^{-1} e_{1} W \\
& \rho u_{0} g_{0} f\left(f I_{0} f\right)^{-1} f e_{1} W=\omega_{L}\left(1 u_{0} g_{0}\right) f e_{1} W,
\end{aligned}
$$

since $I_{0}=R\left(u_{0}\right) g_{0}=e_{1} L\left(u_{1}\right)=L\left(e_{1} u_{1}\right)=L\left(e_{1} W\right)$.

Now these arguments may be applied to $W$ and repeated until (1) is achieved.

The rest of the proof proceeds inductively on the number of segments of $u$ of the form $(q)^{-1}$ with $c(q)=c(u)$.

Suppose $u=p(q)^{-1} r$. By Lemma 3.2, $L(q) L(q)^{-1}(q)^{-1} \rho(q)^{-1}$, so $u \rho p L(q) L(q)^{-1}(q)^{-1} r_{0}$. The $\rho$-class of the word $f(f R(q) q L(q) f)^{-1}$ $f R(q) q L(q)$ is an idempotent in $U / \rho$ that is $\mathscr{L}$-related to $L(q)$, by the same lemma, so

$$
\begin{aligned}
& u \rho p L(q) f(f R(q) q L(q) f)^{-1} f R(q) q L(q) L(q)^{-1}(q)^{-1} r \\
& \rho p L(q) f(f R(q) q L(q) f)^{-1} f R(q) q(q)^{-1} r
\end{aligned}
$$

and thus

$$
u \rho p L(q) f(f R(q) q L(q) f)^{-1} f R(q) r
$$

since $R(q) q(q)^{-1} \rho R(q)$, by the same lemma again. (Compare with [9, Lemma 2.1]).

Note that all the words of the form $f w f$ that appear in (1) or (2) have content $c(u)$. By Theorem 3.3, therefore, each such $(f w f) \rho$ belongs to the subgroup $H_{f \rho}$ of $U / \rho$. Since $f R(L(q)) f$ also belongs to this group,

$$
u \rho p L(q) f(f R(L(q)) f)^{-1}\left(f R(q) q L(q) f(f R(L(q)) f)^{-1}\right)^{-1} f R(q) r .
$$

Suppose $c(p q 0) \neq c(u)$. Then $\langle p q 0\rangle_{q \sigma}=1(p q 0) q \sigma\left(=1 u_{0} g_{0}\right)$ and

$$
\begin{aligned}
\omega_{L}(1(p q 0) q \sigma) & =p q 0 q \sigma f(f R(p q 0) q \sigma f)^{-1} \\
& =p L(q) f(f R(L(q)) f)^{-1}
\end{aligned}
$$

since $L(q)=q 0 q \sigma$ and $R(L(q))=R(q 0 q \sigma)=R(p q 0 q \sigma)=R(p q 0) q \sigma$. 
Otherwise $c(p q 0)=c(u)$. Let $[p q 0]=\left(\pi_{j} f_{j} r_{j} h_{j}\right)_{0 \leq j \leq \ell}$. By the inductive hypothesis,

$$
p q 0 \rho \omega_{L}\left(1 r_{0} h_{0}\right) \prod_{j=1}^{\ell-1} \omega\left(f_{j} r_{j} h_{j}\right)^{\pi_{j}} \omega_{R}\left(f_{\ell} r_{\ell}\right)
$$

Since $c(q 0)=c\left(r_{\ell}\right)$, we have $R(q 0)=R\left(r_{\ell}\right)$ and $R(L(q))=R(q 0) q \sigma=$ $R\left(r_{\ell}\right) q \sigma$.

Hence

$$
\omega_{R}\left(f_{\ell} r_{\ell} 1\right) q \sigma f(f R(L(q)) f)^{-1}=f f_{\ell} r_{\ell} q \sigma f\left(f R\left(r_{\ell}\right) q \sigma f\right)^{-1}=\omega\left(f_{\ell} r_{\ell} q \sigma\right)
$$

and

$$
p L(q) f(f R(L(q)) f)^{-1} \rho \omega_{L}\left(1 r_{0} h_{0}\right) \prod_{j=1}^{\ell-1} \omega\left(f_{j} r_{j} h_{j}\right)^{\pi_{i}} \omega\left(f_{\ell} r_{\ell} q \sigma\right) .
$$

This covers the first segment of the right hand side of (3). We next treat the large inverted term. Let $[(q 1) q(q 0)]=\left(\eta_{j} c_{j} t_{j} d_{j}\right)_{0 \leq j \leq h}$. By the inductive hypothesis,

$$
q 1 q q 0 \rho \omega_{L}\left(1 t_{0} d_{0}\right) \prod_{j=1}^{h-1} \omega\left(c_{j} t_{j} d_{j}\right)^{\eta_{j}} \omega_{R}\left(c_{h} t_{h}\right) \text {. }
$$

Now $f R(q)=f q \epsilon q 1$ and $f q \epsilon \omega_{L}\left(1 t_{0} d_{0}\right)=\omega\left(q \epsilon t_{0} d_{0}\right)$; similarly, $L(q) f$ $(f R(L(q)) f)^{-1}=q 0 q \sigma f(f R(L(q)) f)^{-1}$ and $\omega_{R}\left(c_{h} t_{h} 1\right) q \sigma f(f R(L(q)) f)^{-1}$ $=\omega\left(c_{h} t_{h} q \sigma\right)$, since $R(L(q))=R(q 0) q \sigma=R\left(t_{k}\right) q \sigma$, similarly to the earlier argument. Combining these two equations gives

$$
f R(q) q L(q) f(f R(L(q)) f)^{-1} \rho \omega\left(q \epsilon t_{0} d_{0}\right) \prod_{j=1}^{h-1} \omega\left(c_{j} t_{j} d_{j}\right)^{\eta_{j}} \omega\left(c_{h} t_{h} q \sigma\right) .
$$

A similar, but simpler, analysis applies to the last segment, $f R(q) r$, of (3). By substituting these equations into (3) and comparing the result with the definition of $[u]$, the proof of the lemma is completed.

To complete the proof of sufficiency, let $u, v \in U$, with $|c(u)| \geq 2$, satisfying the conditions in Theorem 4.1. Let $[u]=\left(\mu_{j} e_{j} u_{j} g_{j}\right)_{0 \leq j \leq k},[v]=$ $\left(\eta_{j} f_{j} v_{j} h_{j}\right)_{0 \leq j \leq \ell}$. By Lemma 4.3,

$$
u \rho \omega_{L}\left(1 u_{0} g_{0}\right) \prod_{j=1}^{k-1} \omega\left(e_{j} u_{j} g_{j}\right)^{\mu_{j}} \omega_{R}\left(e_{k} u_{k}\right)
$$


and

$$
v \rho \omega_{L}\left(1 v_{0} h_{0}\right) \prod_{j=1}^{\ell-1} \omega\left(f_{j} v_{j} h_{j}\right)^{\eta_{j}} \omega_{R}\left(f_{\ell} v_{\ell}\right) .
$$

Let $\psi$ be any map of Link $(u) \cup \operatorname{Link}(v)$ into $Z$, as in the statement of the theorem. Then

$$
\prod_{j=1}^{k-1}\left(e_{j} u_{j} g_{j}\right) \psi^{\mu_{j}}=\prod_{j=1}^{\ell-1}\left(f_{j} v_{j} h_{j}\right) \psi^{\eta_{j}} \quad \text { in } F \mathbf{G}_{Z}
$$

For any $e w g \in \operatorname{Link}(u) \cup \operatorname{Link}(v), \omega(e w g)=f e w g f(f R(w) g f)^{-1}$. Thus for any $e w g, e^{\prime} w^{\prime} g^{\prime} \in \operatorname{Link}(u) \cup \operatorname{Link}(v)$, if $(e w g) \psi=\left(e^{\prime} w^{\prime} g^{\prime}\right) \psi$ in $Z$ then $\omega(e w g) \rho \omega\left(e^{\prime} w^{\prime} g^{\prime}\right)$. For by hypothesis $e=e^{\prime}, g=g^{\prime}$ and $w \rho w^{\prime}$, whence, by Lemma 3.1, $R(w) \rho R\left(w^{\prime}\right)$. Since for all such $e w g$, $(e w g) \rho$ belongs to the subgroup $H_{f_{\rho}}$ of $U / \rho$,

$$
\prod_{j=1}^{k-1} \omega\left(e_{j} u_{j} g_{j}\right)^{\mu_{j}} \rho \prod_{j=1}^{\ell-1} \omega\left(f_{j} v_{j} h_{j}\right)^{\eta_{j}} .
$$

But by hypothesis (ii), $u_{0} \rho \quad v_{0}$ and $g_{0}=h_{0}$, so $R\left(u_{0}\right) \rho R\left(v_{0}\right)$ and $\omega_{L}\left(1 u_{0} g_{0}\right) \rho w_{L}\left(1 v_{0} h_{0}\right)$. Similarly $\omega_{R}\left(e_{k} u_{k}\right) \rho \omega_{R}\left(f_{\ell} v_{\ell}\right)$. Hence $u \rho v$.

COROLLARY 4.4. Let $\left\{S_{i}\right\}_{i \in I}$ be a disjoint family of completely simple semigroups and put $S=*_{\mathrm{CR}} S_{i}$. Then the maximal subgroups of $S$, other than those of the original factors $S_{i}$, are free groups.

PROOF. Let $H$ be a maximal subgroup of $S$ that is disjoint from $\bigcup_{i \in l} S_{i}$. By Theorem 3.3, $H$ determines a finite subset $A=\left\{i_{1}, i_{2}, \ldots, i_{n}\right\}$ of $I$, with $n \geq 2$, namely $c(u)$ for any $u \in H$. Let $\operatorname{Link}_{A}=\bigcup\{\operatorname{Link}(u): u \in U, c(u)=A\}$. Let $\psi$ be a map of $\operatorname{Link}_{A}$ into a sufficiently large set $Y$, such that for $e w g$, $e^{\prime} w^{\prime} g^{\prime} \in \operatorname{Link}_{A},(e w g) \psi=\left(e^{\prime} w^{\prime} g^{\prime}\right) \psi$ if and only if $e=e^{\prime}, w \rho w^{\prime}$ and $g=g^{\prime}$.

For $1 \leq j \leq n$, let $s_{i_{j}}$ be the designated idempotent in $S_{i_{j}}$, as in Section 3. Put $s=s_{i_{1}} \ldots s_{i_{n}} s_{i_{1}} \ldots s_{i_{n-1}}$. By Theorem 3.3, $H \subseteq D_{s \rho}$, and so $H \cong H_{s \rho}$. Clearly, $s 0=s_{i_{1}} \ldots s_{i_{n-1}}=s 1$ and $s \sigma=s \epsilon=s_{i_{n}}=d$, say.

Note that

$$
{ }_{d}\langle s 1 s 0\rangle_{d}=d(s 1 s 0) d=d\left(s_{i_{1}} \ldots s_{i_{n-1}}\right)^{2} d \in \operatorname{Link}\left(s_{i_{n}} s_{i_{1}} \ldots s_{i_{n-1}} s_{i_{1}} \ldots s_{i_{n}}\right),
$$

so $(d(s 1 s 0) d) \psi$ is well defined. 
Let $u, v \in U$, with $[u]=\left(\mu_{j} e_{j} u_{j} g_{j}\right)_{0 \leq j \leq k}=\left(1 u_{0} g_{0}, T, e_{k} u_{k} \cdot 1\right)$ and [v] $=\left(\eta_{j} f_{j} v_{j} h_{j}\right)_{0 \leq j \leq \ell}=\left(1 v_{0} h_{0}, V, f_{\ell} v_{\ell} \cdot 1\right)$, say. If $u \rho, v \rho \in H_{s \rho}$ then by Theorem 3.3, $u_{0}=u 0 \rho s 0 \rho v 0=v_{0}, g_{0}=u \sigma=s \sigma=d=v \sigma=h_{0}, u_{k}=$ $u 1 \rho s 1 \rho v 1=v_{\ell}$ and $g_{k}=u \epsilon=s \epsilon=d=v \epsilon=h_{\ell}$. By Lemma 4.2 and its dual $[u v]=\left(1 u_{0} g_{0}, T,{ }_{d}\langle u 1 v 0\rangle_{d}, V, f_{\ell} v_{\ell} 1\right)$.

Now $\left({ }_{d}\langle u 1 v 0\rangle_{d}\right) \psi=(d s 1 s 0 d) \psi$, since $u 1 v 0 \rho s 1 s 0$. Thus the map

$$
u \rho \longmapsto \prod_{j=1}^{k-1}\left(e_{j} u_{j} g_{j}\right) \psi^{\mu_{j}}(d s 1 s 0 d) \psi
$$

is a morphism of $H_{s \rho}$ into $F \mathbf{G}_{Y}$ which, by Theorem 4.1 , is injective. The Schreier subgroup theorem completes the proof.

\section{Coproducts of groups}

Let $\left\{G_{i}\right\}_{i \in I}$ be a family of disjoint groups. Theorem 4.1 simplifies, to the extent that the solution looks almost identical to that for free completely regular semigroups. For the designated idempotent $s_{i}$ of $G_{i}$ is obviously its identity, and $H^{(i)}=L^{(i)}=R^{(i)}=G_{i}$. Hence if $u \in U=*_{\mathrm{U}} G_{i}$ and $e_{j} u_{j} g_{j}$ is a link of $u$, then either $e_{j}=1$ or $e_{j}$ is the identity of $G_{i_{j}}$, where $c(u)-c\left(u_{j}\right)=\left\{i_{j}\right\}$, and similarly for $g_{j}$. Thus we may omit mention of $e_{j}$ and $g_{j}$ altogether. Theorem 3.3 now specializes as follows.

PROPOSITION 5.1. Let $\left\{G_{i}\right\}_{i \in I}$ be a family of disjoint groups, $U=*_{\mathrm{U}} G_{i}$ and $S=U / \rho=*_{\mathrm{CR}} G_{i}$. If $u, v \in U$ then

(i) $u \rho \mathscr{D} v \rho$ if and only if $c(u)=c(v)$;

(ii) $\quad u \rho \mathscr{R} v \rho$ if and only if $c(u)=c(v)$ and $u 0 \rho v 0$;

(iii) $u \rho \mathscr{L} v \rho$ if and only if $c(u)=c(v)$ and $u 1 \rho v 1$.

The definition of the characteristic sequence $[u]$ of $u$ may be simplified: $[u]=\left(\mu_{j} u_{j}\right)_{0 \leq j \leq k}$, where

(i) if $u$ has no segment of the form $(q)^{-1}$ with $c(q)=c(u)$, then $\mu_{j}=1$ and $u_{j}=\hat{b}_{j}, 0 \leq j \leq k$, as before, and

(ii) if $u=p(q)^{-1} r$, where $c(q)=c(u)$, then $[u]=(\langle p q 0\rangle,-[q 1 q q 0]$, $\langle q 1 r\rangle)$, where

$$
\langle w\rangle= \begin{cases}w & \text { if } c(w) \neq c(u) \\ {[w]} & \text { if } c(w)=c(u)\end{cases}
$$


Now Theorem 4.1 becomes the following (cf. [8, Theorem]).

COROLLARY 5.2. Let $\left\{G_{i}\right\}_{i \in I}$ be a family of disjoint groups and let $U=*_{\mathrm{U}} G_{i}$. Let $u, v \in U,|c(u)| \geq 2$. Then $u \rho v$ if and only if

(i) $\quad c(u)=c(v)$,

(ii) $u 0 \rho v 0$,

(iii) $u 1 \rho v 1$,

(iv) if $[u]=\left(\mu_{j} u_{j}\right)_{0 \leq j \leq k}$ and $[v]=\left(\eta_{j} v_{j}\right)_{0 \leq j \leq \ell}$ and $\psi$ is any map from $\left\{u_{1}, \ldots, u_{k-1}\right\} \cup\left\{v_{1}, \ldots, v_{\ell-1}\right\}$ to $Z$ such that for $s, t$ in the domain, $s \psi=t \psi$ if and only if $s \rho t$, then $\prod_{j=1}^{k-1}\left(u_{j} \psi\right)^{\mu_{j}}=\prod_{j=1}^{\ell-1}\left(v_{j} \psi\right)^{\eta_{j}}$ in $F \mathbf{G}_{Z}$.

By standard arguments, when each group $G_{i}$ is infinite cyclic, $*_{\mathbf{C R}} G_{i} \cong$ $F \mathrm{CR}_{I}$ and Corollary 5.1 becomes precisely the Theorem in [8]. A more novel special case is that when each group $G_{i}$ is trivial, $G_{i}=\left\{e_{i}\right\}$, say. Let $\bar{I}=\left\{e_{i}\right.$ : $i \in I\}$. Then $\bar{I}$ is a set of idempotents of $S=*_{\mathrm{CR}}\left\{e_{i}\right\}$ which generates $S$ and is bijective with $I$.

PROPOSITION 5.3. The free product $S=*_{\mathrm{CR}}\left\{e_{i}\right\}$ of trivial groups $\left\{e_{i}\right\}_{i \in I}$, together with the injection $\theta: i \mapsto e_{i}, i \in I$, is the free idempotent-generated completely regular semigroup $F I \mathbf{C R}_{I}$ on $I$, in the following sense. For any map $\phi$ of $I$ into the set $E_{T}$ of idempotents of a completely regular semigroup there is a unique morphism $\bar{\phi}: S \rightarrow T$ such that $\theta \bar{\phi}=\phi$.

PROOF. Let $\phi$ be as in the statement of the theorem. Then for each $i$, the restriction $\phi_{i}$ of $\phi$ to $\{i\}$ induces the monomorphism $\bar{\phi}_{i}$ of the group $\left\{e_{i}\right\}$ into $T$ given by $e_{i} \bar{\phi}_{i}=i \phi$. These monomorphisms extend uniquely to a morphism $\bar{\phi}: S \rightarrow T$ such that $e_{i} \bar{\phi}=e_{i} \bar{\phi}_{i}$ for all $i \in I$. Thus $\theta \bar{\phi}=\phi$.

Corollary 5.2 therefore solves the word problem in $F I \mathbf{C R}_{A}$, for any set $A$. In particular, by Corollary 4.4 , the nontrivial subgroups of $F I \mathbf{C R}_{A}$ are free. In the next section we show that $F I \mathbf{C R}_{A}$ is isomorphic with the completely regular subsemigroup of $F \mathbf{C R}_{A}$ generated by $\left\{a^{0}: a \in A\right\}$.

\section{Subsemigroups of the free product}

In this section we show that if for each $i \in I, T_{i}$ is a (completely) regular subsemigroup of the completely simple semigroup $S_{i}$ then the completely regular 
subsemigroup of the free product $S=*_{\mathrm{CR}} S_{i}$ generated by $\bigcup_{i \in I} T_{i}$ is isomorphic to $*_{\mathrm{CR}} T_{i}$. (It was shown in [7] that for bands, at least, this need not happen if complete simplicity of the factors is relaxed. We do not know if the same is true for completely regular semigroups.)

Recall from Section 1 the notation $X$, for $\bigcup_{i \in I} S_{i}$, and $F$, for the free monoid on $X \cup\left\{(,)^{-1}\right\}$. Now let $Y=\bigcup_{i \in I} T_{i} \subseteq X$ and let $F_{Y}$ be the submonoid of $F$ generated by $Y \cup\left\{(,)^{-1}\right\}$. Clearly, $F_{Y}$ is freely generated by this set. Also $F \mathbf{U}_{Y}$, the free unary semigroup on $Y$, is (isomorphic to) the smallest subsemigroup of $F_{Y}$ such that $Y \subseteq F \mathbf{U}_{Y}$ and $(w)^{-1} \in F \mathbf{U}_{Y}$ whenever $w \in F \mathbf{U}_{Y}$; thus $F \mathbf{U}_{Y}$ is the unary subsemigroup of $F \mathbf{U}_{X}$ generated by $Y$. Let $\epsilon_{Y}$ denote the unary congruence on $F \mathbf{U}_{Y}$ generated by

$$
\left\{(s \cdot t, s t): s, t \in T_{i}, i \in I\right\} \cup\left\{\left((t)^{-1}, t^{-1}\right): t \in T_{i}, i \in I\right\} .
$$

Suppose $v, w \in F \mathbf{U}_{Y}$ and $v \epsilon w$ (in $F \mathbf{U}_{X}$ ). Let $\bar{v}$ and $\bar{w}$, respectively, denote the unique words in $F \mathbf{U}_{Y}$ that are $\epsilon_{Y}$-related to $v$ and $w$ according to Proposition 1.2, as applied to $Y$. Then applying the same proposition to $X$ we see that since $\epsilon_{Y} \subseteq \epsilon_{X}, \bar{v}=\bar{w}$ and so $v \epsilon_{Y} w$. Hence $\epsilon$ restricts to $\epsilon_{Y}$ on $F \mathbf{U}_{Y}$ and we may identify the free unary product $*_{\mathrm{U}} T_{i}$ with a unary subsemigroup $U_{Y}$, say, of $U=$ $*_{\mathrm{U}} c p S_{i}$. Furthermore, we may suppose that $U_{Y}$ comprises those 'reduced'words of $U$ whose letters (other than ( and $)^{-1}$ ) belong to $Y$ (see the remarks following Proposition 1.2).

All the discussion so far has been valid in general, for $T_{i}$ a unary subsemigroup of $S_{i}, i \in I$. Now let each $S_{i}$ be completely simple. Denote by $\rho_{Y}$ the congruence on $U_{Y}$ that induces $*_{\mathrm{CR}} T_{i}$, as in Proposition 1.3. In the notation of Section 3, for each $i \in I$, we may choose the designated idempotent $s_{i}$ of $S_{i}$ from $T_{i}$. For $u \in U$, if $u \in U_{Y}$ it is clear that $u 0, u 1 \in U_{Y}$ also. Further, $u \sigma=\left(y s_{j}\right)^{0} \in U_{Y}$ and $u \epsilon=\left(s_{j} x\right)^{0} \in U_{Y}$ (see Section 3 for the definition of $x$ and $y$ ). In fact, if $[u]=\left\{\mu_{j} e_{j} u_{j} g_{j}\right\}_{0 \leq j \leq k}$ denotes the characteristic sequence (Section 4) of $u$, regarded as an element of $U$, then similar reasoning shows that each $e_{j}, g_{j} \in T_{i_{j}}$ for some $i_{j} \in I$, and each $u_{j} \in U_{Y}$. Hence $[u]$ is also the characteristic sequence of $u$, when $u$ is regarded as an element of $U_{Y}$.

Now denote by $\left\langle\bigcup_{i \in I} T_{i}\right\rangle$ the completely regular subsemigroup of $S=*_{\mathrm{CR}} S_{i}$ generated by the subsemigroups $T_{i}, i \in I$. Clearly, $\left\langle\bigcup_{i \in I} T_{i}\right\rangle$ is the image of $U_{Y}$ under the projection of $U$ on $U / \rho=S$. From Theorem 4.1 and the discussion in the preceding paragraph, it is now evident that for words $u, v$ in $U$, if $u, v \in U_{Y}$ and $u \rho v$ then $u \rho_{Y} v$; that is, $\rho$ restricts to $\rho_{Y}$ on $U_{Y}$. Hence $\left\langle\bigcup_{i \in I} T_{i}\right\rceil \cong *_{\mathbf{C R}} T_{i}$. This completes the proof of the main result of the section. 
THEOREM 6.1. Let $\left\{S_{i}\right\}_{i \in I}$ be a family of completely simple semigroups and let $T_{i}$ be a regular subsemigroup of $S_{i}, i \in I$. Then the unary subsemigroup of $*_{\mathrm{CR}} S_{i}$ generated by the semigroups $T_{i}, i \in I$, is isomorphic with $*_{\mathrm{CR}} T_{i}$.

COROLLARY 6.2. The free idempotent-generated completely regular semigroup $F I \mathbf{C R}_{A}$ on a set $A$ is isomorphic with the unary subsemigroup of $F \mathbf{C R}_{A}$ generated by $\left\{a^{0}: a \in A\right\}$.

\section{References}

[1] A. H. Clifford, 'The free completely regular semigroup on a set', J. Algebra 59 (1979), 434-451.

[2] A. H. Clifford and G. B. Preston, The algebraic theory of semigroups, vol. I (Amer. Math. Soc., Providence, Rhode Island, 1961).

[3] J. A. Gerhard, 'Free completely regular semigroups I and II', J. Algebra 82 (1983), 135-142 and $143-156$.

[4] G. Grätzer, Universal algebra (Springer, New York, 1979).

[5] J. M. Howie, An introduction to semigroup theory (Academic Press, London, 1976).

[6] P. R. Jones, 'Completely simple semigroups: free products, free semigroups and varieties', Proc. Royal Soc. Edinburgh Sect. A 88 (1981), 293-313.

[7] — 'On free products of bands', Semigroup Forum 43 (1991), 53-62.

[8] J. Kadourek and L. Polák, 'On the word problem for free completely regular semigroups', Semigroup Forum 34 (1986), 127-138.

[9] P. G. Trotter, 'Free completely regular semigroups', Glasgow Math. J. 25 (1984), 241-254.

Department of Mathematics, Statistics and Computer Science

Marquette University

Milwaukee, WI 53233

USA 